\title{
Retos de la entomología médica para la vigilancia en salud pública en Colombia: reflexión para el caso de malaria
}

\author{
Helena Brochero ${ }^{1}$, Martha L. Quiñones ${ }^{2}$ \\ ${ }_{1}^{1}$ Área de Entomología, Facultad de Agronomía, Universidad Nacional de Colombia, Bogotá D.C., Colombia \\ ${ }^{2}$ Departamento de Salud Pública, Facultad de Medicina, Universidad Nacional de Colombia, Bogotá D.C., \\ Colombia
}

\begin{abstract}
Se presenta una reflexión sobre la pertinencia del fortalecimiento de la vigilancia entomológica en los programas de control de paludismo en un escenario como Colombia, haciendo un reconocimiento de los vacíos de información sobre el conocimiento de las especies vectores y resaltando las acciones básicas que requiere el país en este campo. Bajo la premisa que los programas de control de vectores deben estar basados en resultados de los estudios entomológicos locales y dar respuestas prácticas a preguntas orientadas al control de la enfermedad, se mencionan algunas dificultades por resolver con relación a las especies de Anopheles en Colombia, particularmente en lo que tiene que ver con la taxonomía e incriminación como vectores y los retos que se podrían asumir en este aspecto.
\end{abstract}

Palabras clave: entomología, vigilancia de la población, malaria/prevención y control, Anopheles, salud pública.

Challenges of the medical entomology for the surveillance in public health in Colombia: reflections on the state of malaria

The relevance of the medical entomology was considered with respect to current framework of malaria control programs in Colombia. A responsibility is indicated for balancing control efforts along with providing information on the malaria vectors. This knowledge must be acquired in order to focus the related activities that are required. The malaria control program must be based on results of local entomological surveillance, and the data must be in a form to give practical answers to questions regarding the control program. Difficulties in undertaking the required studies are described, particularly regarding the taxonomic identification of Colombian Anopheles in Colombia and which of these can be incriminated as malaria vectors.

Key words: entomology, population surveillance, malaria/prevention \& control, Anopheles, public health.

En la última década, a través del Ministerio de Protección Social y del Instituto Nacional de Salud, se ha venido promoviendo en Colombia, la estructuración y consolidación de una Red de Entomología Médica como apoyo al Programa Nacional de Enfermedades Transmitidas por Vectores. La red tiene su sustento a nivel

Correspondencia:

Helena Luisa Brochero, Facultad de Agronomía, Universidad Nacional de Colombia,

edificio 500, campus universitario, avenida carrera $30 \mathrm{~N}^{\circ}$. 45-03, Bogotá D.C., Colombia. Tel: (571) 3165000 ext. 19013 embrochero@unal.edu.co

Recibido: 29/05/07; aceptado: 13/10/07 departamental a partir de la conformación de unidades de entomología coordinadas por profesionales formados en entomología médica. El papel de los entomólogos, articulados en una discusión multidisciplinaria permanente, conjuntamente con las áreas de laboratorio (virología, parasitología, patología), epidemiología, gestión administrativa, y desde una visión intrasectorial e intersectorial, se hace indispensable, particularmente cuando las medidas de control dirigidas a los vectores parecen no funcionar y el número de casos de las enfermedades se incrementa. En este sentido, las unidades de entomología deben proveer 
información actualizada sobre las especies vectores presentes, su comportamiento, distribución, susceptibilidad a insecticidas, efectividad y eficacia de las medidas de control realizadas a nivel local en cada región. Esta información es definitiva para focalizar, priorizar, evaluar y replantear las estrategias de control. Presentamos una reflexión sobre algunos vacíos que se tienen sobre el conocimiento de las especies vectores resaltando las acciones básicas que requiere el país en este campo de la entomología médica con un enfoque para el control del paludismo.

El control de malaria se basa, además de la atención adecuada y oportuna de los pacientes, en medidas de control dirigidas a sus vectores. La Organización Mundial de la Salud (OMS) define que las actividades de la entomología médica son esenciales para "orientar adecuadamente las estrategias de control integrado $y$ selectivo de vectores" (1). En este sentido indica que en los programas de malaria, los principales propósitos de los estudios entomológicos son disponer de la información necesaria para planear las acciones de control de la enfermedad y evaluar la efectividad de estas medidas (1). En algunos casos particulares se recomiendan acciones en los criaderos para disminuir las densidades de mosquitos.

Sin embargo, la medida de elección para el control es la aplicación de insecticidas residuales en las viviendas, bien sea en las paredes o en los toldillos, con el propósito de lograr una reducción en la longevidad de los vectores debido a la mortalidad de una fracción de la población de mosquitos hembra que entra en contacto con el insecticida (2). Con el uso de toldillos impregnados, se logra además, disminuir el contacto humanovector por la barrera física que constituye. Algunas veces se puede notar una reducción en la densidad de las poblaciones de vectores, tanto con la aplicación de insecticidas en las paredes como en los toldillos, aunque el objetivo es interrumpir el ciclo de transmisión a través de la disminución de la longevidad de la población de vectores. Si el promedio de días que sobrevive la población de mosquitos se reduce, será menor la proporción de anofelinos capaces de permitir el desarrollo de los parásitos y ser infectivos en su picadura a los humanos.

Paralelamente, es necesario adelantar estudios epidemiológicos y entomológicos a nivel local teniendo en cuenta que las características de la transmisión del paludismo presentan diferencias según la región, lo mismo que los hábitos de los vectores, sus sitios de reposo, la actividad de picadura, el grado de antropofilia y la respuesta a la presión de los insecticidas. En un país con una diversidad ecológica tan grande como Colombia, la complejidad es evidente.

De la antes considerada única medida para erradicar la malaria a nivel mundial promovida por la OMS en la década de los 60 , basada en la aplicación de insecticidas residuales, se pretende ahora desarrollar un control "selectivo e integrado". Este enfoque implica un mayor conocimiento de la epidemiología de la enfermedad a nivel local y toma de decisiones acordes a cada situación en particular, por lo que se hace aún más relevante el papel de la entomología médica en los programas de control de malaria.

La OMS menciona como responsabilidades básicas de las unidades de entomología médica en los servicios departamentales de salud: 1) identificar los vectores responsables de la transmisión; esto incluye tener claridad en su taxonomía e incriminar las especies vectores; 2 ) conocer los hábitos y hábitats de las especies vectores; 3) participar en la selección de las medidas de control que se deben implementar, según los hábitos y características de las especies vectores; 4) evaluar el impacto de las medidas de control, determinando, según sea el caso, cambios en la longevidad, densidad, tasas de infección, susceptibilidad de los vectores a los insecticidas y duración del efecto residual en las superficies tratadas con insecticidas, y 5) participar en la focalización y priorización de áreas geográficas para la ejecución de las acciones de control.

En particular, dar respuesta a "áreas problema", es decir, cuando estando bien aplicadas las medidas de control, con la suficiente cobertura y periodicidad, el número de casos de malaria continúa igual o se incrementa $(1,3)$. Se entiende, entonces, que los programas de control de 
vectores deben estar sustentados en resultados de los estudios entomológicos locales y deben dar respuestas prácticas a preguntas orientadas al control de la enfermedad. De hecho, la vigilancia entomológica no se limita a actividades de control solamente, sino que también suministra información básica para el entendimiento de la epidemiología de la enfermedad. Por ejemplo, debido a la diversidad de especies de Anopheles presentes en Colombia, es necesario disponer de información actualizada propia de cada especie. Se debe entender que las especies son organismos dinámicos, complejos, en permanente adaptación, por lo que es necesario actualizar permanentemente el conocimiento que se tiene de ellas. Para definir y orientar adecuadamente las medidas de control es básico conocer cuáles especies se encuentran presentes en la región y cuáles de ellas se encuentran actuando como vectores.

A continuación se ilustran algunas dificultades por resolver con relación a las especies de Anopheles con distribución en Colombia, particularmente en lo que tiene que ver con la incriminación como vectores. El indagar sobre estos aspectos constituye el primer paso esencial para diseñar adecuadamente cualquier estrategia de control.

Con base en el aislamiento de parásitos Plasmodia de humanos y la información epidemiológica, Colombia reconoce como vectores primarios de malaria a Anopheles (Nyssorhynchus) darlingi Root 1926, A. (Nys.) albimanus Wiedemann 1820 y A. (Nys) nuneztovari Gabaldon $1940(4,5)$. Como vectores secundarios se consideran $A$. (Anopheles) pseudopunctipennis Theobald 1901, A. (An) punctimacula Dyar \& Knab 1906, A. (Kertezsia) neivai Howard, Dyar \& Knab $1912(4,5)$ y $A$. (K) lepidotus Zavortink 1973 (6). Hacia estas entidades biológicas se ha centrado la vigilancia entomológica y las acciones de control integrado y selectivo de vectores se han dirigido a estas especies. En un país de tanta riqueza ecogeográfica y con pluralidad en el desarrollo territorial de sus departamentos, uno podría preguntarse, si la participación de estas especies y de otros anofelinos registrados en Colombia, es estática y puede ser asumida como un todo generalizado a nivel nacional.
Haciendo una breve revisión, se encuentra que países limítrofes incriminan como transmisores de malaria a especies de Anopheles presentes en Colombia pero que no se consideran vectores en nuestro país. A. aquasalis Curry 1932 es calificado como vector primario, además de $A$. darlingi y $A$. nuneztovari en Venezuela (7). En Brasil, A. marajoara Galvão \& Damasceno 1942 juega un papel tan importante en la transmisión de malaria como $A$. darlingi $(8,9)$ y en algunas regiones como Amapá, A. oswaldoi Peryassu 1922 se reconoce como vector principal (10). Ecuador considera a $A$. rangeli Gabaldon, Cova García \& López, 1940 y A. pseudopunctipennis junto con A. darlingi como vectores principales (11), mientras que $A$. benarrochi Gabaldon, García \& López 1941, A. trinkae Faran 1979, A. triannulatus Neiva \& Pinto 1922 y $A$. darlingi, son reconocidos como responsables de la transmisión de malaria en Perú $(12,13)$. El único denominador común es A. darlingi, y varios estudios avalan la tendencia antropofilica y la capacidad vectorial de esta especie (14-16). Esta situación se torna particularmente preocupante en las áreas limítrofes entre los países, en donde las especies son las mismas independientemente de las fronteras geográficas, pero las consideraciones sobre cuáles de éstas son vectores y las acciones de control siguen los lineamientos particulares de cada país.

Para agravar esta situación, a partir de los avances de la entomología molecular se ha hecho evidente que en la región neotropical, los mosquitos del género Anopheles constituyen complejos de especies debido a las relaciones filogenéticas cercanas entre sus taxones y a la presencia de especies crípticas determinadas por la similaridad interespecífica y la variación intraespecífica de sus caracteres morfológicos. Por ejemplo, en el área de frontera de Colombia con Ecuador y Perú, $A$. noroestensis Galvão \& Lane 1937, con el sinónimo de $A$. evansae Brethes 1926, fue la especie predominante e incriminada como transmisor de malaria en las décadas de los 70 y 80 (17). Sin embargo, un estudio en el departamento del Putumayo reveló que el mosquito con mayor abundancia picando humanos no correspondía a $A$. evansae sino a una especie por describir dentro del complejo $A$. benarrochi, 
señalada inicialmente como una "variante morfológica" denominada $A$. benarrochiB $(18,19)$. Además, se encontraron naturalmente infectadas con parásitos Plasmodia humano, a $A$. rangeli y una nueva especie putativa denominada $A$. oswaldoi B (20-22). De forma similar, se ha encontrado que $A$. punctimacula y $A$. pseudopunctipennis incluyen especies crípticas que forman complejos de especies con diferencias importantes en sus patrones de comportamiento y en sus caracteres morfológicos (23-25). A. punctimacula presenta caracteres morfológicos muy cercanos con A. calderoni Wilkerson 1991 y A. malefactor Dyar \& Knab 1907, de las cuales no existen hasta el momento registros en nuestro país (26). De esta manera, es difícil determinar cuál de estas especies realmente se encuentra en Colombia y, por tanto, la determinación de su distribución, comportamiento de picadura, sitios de cría y capacidad vectorial y, por ende, su importancia como transmisores de paludismo sería necesario definirla mediante estudios locales.

Lounibos y Conn (2000) proponen que existen anofelinos, como $A$. darlingi, que presentan una amplia distribución geográfica y reconocido papel como eficientes vectores de malaria, pero que otras especies pueden estar actuando como vectores locales o regionales manteniendo las endemias de la enfermedad al coexistir con los vectores primarios (27). Tal es el caso de $A$. marajoara reconocido vector principal de malaria en el nororiente de la amazonia brasilera (8). Este mosquito pertenece al complejo Albitarsis constituido además por $A$. albitarsis sensu stricto Lynch-Arribalzaga 1878, A. deaneorum RosaFeitas $1989 \mathrm{y}$, por lo menos, tres especies putativas denominadas $A$. albitarsis $\mathrm{B}, A$. albitarsis $\mathrm{E}$ y más recientemente, $A$. albitarsis $\mathrm{F}$ (28-31). Las hembras de este complejo sólo pueden ser diferenciadas por técnicas de biología molecular $(31,32)$. Además, $A$. marajoara ha sido conocido históricamente como Anopheles albitarsis ss (especie actualmente considerada como restringida en el semitrópico al sur de Argentina); como A. allopha Peryassu 1921 denominación actualmente considerada como nomen dubium; y también como A. domesticus Galvão \& Damasceno 1944, la cual se le consideró como un sinónimo.
Colombia fue adoptando estas diferentes denominaciones taxonómicas, sin mediar necesariamente, estudios entomológicos locales. En el país, A. marajoara presenta una amplia distribución en simpatría con $A$. darlingi (33), se ha encontrado naturalmente infectada por $P$. falciparum (34), exitosamente infectada por $P$. vivax (35), con niveles de resistencia a DDT (36), bien adaptada a cambios bruscos hechos por el hombre (33) y con una estructura genética similar a la de vectores primarios de malaria (37). No obstante lo enunciado, y el registro de $A$. albitarsis F en simpatría con $A$. marajoara para Colombia (38), el país no considera esta especie como de importancia en salud pública. En otro ejemplo similar, Colombia aceptó el cambio en la denominación de $A$. mediopunctatus Theobald 1903 por A. forattini Wilkerson \& Sallum, 1999 o A. costai Fonseca \& Silva Ramos 1939 con base en estudios llevados a cabo por Sallum y colaboradores y por Wilkerson y Sallum en 1999, los cuales incluyeron muy pocas muestras de nuestro país $(39,40)$. Estas denominaciones no han sido incluidas en las claves taxonómicas de mosquitos que utiliza el país para la obtención de inventarios de especies, distribución y vigilancia en salud pública, de forma que no es posible definir realmente si se encuentran en Colombia o si no lo están.

Es evidente, entonces, que tenemos una realidad entomológica para malaria muy compleja. La vigilancia entomológica, como componente fundamental de la salud pública, debe responder a preguntas y necesidades prácticas para el control de las enfermedades transmitidas por vectores, entre otras: ¿cuáles son las especies vectores en su región?, ¿cómo es su comportamiento y hábitos de picadura?, ¿cuándo es pertinente aplicar las medidas de control?, ¿cuál método de control es pertinente ante las situaciones locales particulares: control químico, biológico, social, físico?

Es necesario enfocar hacia la eficacia de la intervención, cuantificar el impacto sobre las poblaciones de vectores y evaluar por cuánto tiempo funcionan apropiadamente las estrategias, para definir, entre otros, la frecuencia de aplicación. También es necesario vigilar la resistencia de los 
vectores a los productos aplicados, los cambios en el comportamiento de los vectores y detectar problemas operativos durante la aplicación. En este contexto, es importante entender la complejidad y la dinámica permanente de los vectores, tener conocimiento sobre la sistemática, filogenia, taxonomía y las interrelaciones con la bionomía de las especies en el marco cambiante de su nicho ecológico. El entendimiento de estos aspectos es insumo fundamental para obtener mapas de distribución geográfica, de incriminación vectorial, de características regionales a través de una vigilancia entomológica regular, sostenida y sistemática, y esta información es esencial para orientar adecuadamente las estrategias de control integrado y selectivo de vectores. En esta tarea, las unidades locales de entomología tienen un papel protagónico, pero la responsabilidad debe ser compartida entre los diferentes profesionales en entomología médica del país, independientemente de su filiación laboral. Los institutos, centros de investigación y la academia deben participar en estos procesos, con responsabilidad social y poner al servicio de la salud pública sus habilidades y fortalezas.

Esta reflexión constituye un muy breve referente para la vigilancia de malaria. Sin embargo, Colombia comprende regiones donde actúan concomitantemente diferentes enfermedades transmitidas por vectores como leishmaniosis, dengue, fiebre amarilla, enfermedad de Chagas, encefalitis equina venezolana y encefalitis equina del este, entre otras. Cada departamento requiere un grupo de entomología conformado por profesionales formados en entomología y técnicos capacitados, apoyados en una infraestructura e insumos mínimos para el abordaje de retos fascinantes como los que aquí se enuncian. Estos grupos deben estar sustentados en una normatividad que permita continuidad en las unidades de entomología, de forma tal que la capacitación y la experiencia de los entomólogos y sus técnicos sea de beneficio creciente a los programas de control de las enfermedades transmitidas por vectores. Debe ser un grupo articulado en el marco de los Programas de Enfermedades Transmitidas por Vectores, priorizado por el nivel departamental y asesorado técnicamente por el nivel central como lo propone la Red Nacional de Entomología Médica. Esta red debe también estar asesorada por expertos adscritos a centros de investigación y universidades nacionales e internacionales, en una dinámica permanente de discusión y análisis en concordancia con los nuevos hallazgos que en materia de vectores de malaria se estén adelantando en el neotrópico.

\section{Conflicto de intereses}

Los autores declaramos que no existen conflictos de intereses que puedan afectar los conceptos emitidos en este manuscrito.

\section{Financiación}

Para la elaboración de este ensayo se dispuso de tiempo en la jornada laboral de los autores como docentes en la Universidad Nacional de Colombia.

\section{Referencias}

1. World Health Organization. Malaria entomology and vector control. Learner's guide. Geneva: WHO; 2000. p.107.

2. Najera J, Zaim M. Malaria vector control: Decisionmaking criteria and procedures for judicious use of insecticides. Document WHO/CDS/WHOPES/2002.5. Geneva: WHO; 2002.p.116.

3. Bruce-Chwatt L. Essential malariology. London: William Heinemann Medical Books Ltd.; 1980. p.354.

4. Servicio de Erradicación de la Malaria. Plan de Erradicación de la Malaria en Colombia. Volumen I y II. Bogotá: Ministerio de Salud Nacional; 1957.

5. Olano V, Brochero H, Sáenz R, Quiñones M, Molina J. Mapas preliminares de la distribución de Anopheles vectores de malaria en Colombia. Biomédica. 2001;21:402-3.

6. Quiñones $M L$, Suárez MF, Rodríguez A, Fleming GA, Galvis LE. Comportamiento de Anopheles (Kerteszia) lepidotus Zavortink, 1973, y su incriminación como posible vector de malaria en el departamento del Tolima, Colombia. Biomédica. 1984;4:5-13.

7. Rubio-Palis Y. Anopheles (Nyssorhynchus) de Venezuela: taxonomía, bionomía, ecología e importancia médica. Maracay: Escuela de Malariologia y Saneamiento Ambiental Editores; 2000. p.120.

8. Conn JE, Wilkerson RC, Segura MN, De Souza RT, Schlichting CD, Wirtz $\mathbf{R}$ et al. Emergence of a new neotropical malaria vector facilitated by human migration and land use. Am J Trop Med Hyg. 2002;66:18-22.

9. Povoa MM, de Souza RT, Lacerda RN, Rosa ES, Galiza D, de Souza JR et al. The importance of 
Anopheles albitarsis E and An. darlingi in human malaria transmission in Boa Vista, state of Roraima, Brazil. Mem Inst Oswaldo Cruz. 2006;101:163-8.

10. Povoa M, Wirtz R, Lacerda R, Miles M, Warhurst D. Malaria vectors in the municipality of Serra do Navio, State of Amapá, Amazon Region, Brazil. Mem Inst Oswaldo Cruz. 2001;96:179-84.

11. Pan American Health Organization. Status of malaria programs in the Americas. XLII Report. Washington, D.C.: PAHO; 1994. p.40.

12. Hayes J, Calderón G, Falcon R, Zambrano V. Newly incriminated Anopheles vectors of human malaria parasites in Junin Department, Perú. J Am Mosq Control Assoc. 1987;3:418-22.

13. Aramburú Guarda J, Ramal Asayag C, Witzig R. Malaria re-emergence in the Peruvian Amazon region. Emerg Infect Dis. 1999;5:209-15.

14. Bharti AR, Chuquiyauri R, Brouwer KC, Stancil J, Lin J, Llanos-Cuentas A, et al. Experimental infection of the neotropical malaria vector Anopheles darlingi by human patient-derived Plasmodium vivax in the Peruvian Amazon. Am J Trop Med Hyg. 2006;75:610-6.

15. Flores-Mendoza C, Fernandez R, Escobedo-Vargas K, Vela-Perez Q, Schoeler G. Natural Plasmodium infections in Anopheles darlingi and Anopheles benarrochi (Diptera: Culicidae) from Eastern Peru. J Med Entomol. 2004;41:489-94.

16. Tadei WP, DutaryThatcher B. Malaria vectors in the Brazilian Amazon: Anopheles of the subgenus Nyssorhynchus. Rev Inst Med Trop S Paulo 2000;42:87-94.

17. Ferro C. Revisión de los recursos aplicables a la lucha contra el paludismo. Revista de la Escuela Nacional de Salud Publica de Colombia. 1979;5:11-8.

18. Quiñones ML, Linton Y, Harbach R, Ruiz J, Calle D, Estrada $\mathbf{D}$ et al. Incriminación de vectores de malaria en el sur de Colombia: determinación de especies y papel vectorial. Bogotá: Vélez \& Quiñones Editores, Cátedra Litográfica; 2004. p.31-47.

19. Quiñones ML, Harbach RE, Calle DA, Ruiz F, Erazo HF, Linton YM. Variante morfológica de adultos hembras de Anopheles benarrochi (Diptera:Culicidae) en Putumayo, Colombia. Biomédica. 2001;21:351-9.

20. Ruiz F, Quiñones ML, Erazo HF, Calle DA, Alzate JF, Linton YM. Molecular differentiation of Anopheles (Nyssorhynchus) benarrochi and An (N.) oswaldoi from southern Colombia. Mem Inst Oswaldo Cruz. 2005;100:155-60.

21. Quiñones ML, Ruíz F, Calle DA, Harbach RE, Erazo HF, Linton YM. Incrimination of Anopheles (Nyssorhynchus) rangeli and An. (Nys.) oswaldoi as natural vectors of Plasmodium vivax in Southern Colombia. Mem Inst Oswaldo Cruz. 2006;101:617-23.
22. Marrelli MT, Malafronte RS, Flores-Mendoza C Lourenço-De-Oliveira R, Kloetzel JK, Marinotti 0. Sequence analysis of the second internal transcribed spacer of ribosomal DNA in Anopheles oswaldoi (Diptera: Culicidae). J Med Entomol. 1999;36:679-84.

23. Estrada-Franco JG, Ma MC, Lanzaro GC, Gwadz R, Galvan-Sanchez C, Céspedes J, et al. Evidencia genética de un complejo de especie en Anopheles pseudopunctipennis pseudopunctipennis: Bol Oficina Sanit Panam. 1992;113:297-9.

24. Estrada-Franco JG, Lanzaro GC, Ma MC, WalkerAbbey A, Romans P, Galvan-Sanchez C, et al. Characterization of Anopheles pseudopunctipennis sensu lato from three countries of Neotropical America from variation in allozymes and ribosomal DNA. Am J Trop Med Hyg.1993:49:735-45.

25. Estrada-Franco JG, Ma MC, Gwadz RW, Sakai R, Lanzaro GC, Laughinghouse A, et al. Evidence through crossmating experiments of a species complex in Anopheles pseudopunctipennis sensu lato a primary malaria vector of the American continent. Am J Trop Med Hyg. 1993;49:746-55.

26. Wilkerson RC. Redescriptions of Anopheles punctimacula and Anopheles malefactor (Diptera: Culicidae). J Med Entomol. 1990;27:225-47.

27. Lounibos LP, Conn JE. Malaria vector heterogeneity in South America. Am Entomol. 2000;46:238-49.

28. Wilkerson R, Gaffigan T, Bento-Lima J. Identification of species related to Anopheles (Nyssorhynchus) albitarsis by random amplified polymorphic DNA polymerase chain reaction (Diptera: Culicidae). Mem Inst Oswaldo Cruz. 1995;90:721-32.

29. Rosa-Freitas MG. Anopheles (Nyssorhynchus) deaneorum: a new species in the Albitarsis complex (Diptera: Culicidae). Mem Inst Oswaldo Cruz. 1989;84:535-43

30. Lehr M, Kilpatrick C, Wilkerson R, Conn J. Cryptic species in the Anopheles (Nyssorhynchus) albitarsis (Diptera: Culicidae) Complex: incongruence between RAPD-PCR identification and analysis of mtDNA COI gene sequences. Ann Entomol Soc Am. 2005;98:908-17.

31. Wilkerson RC, Parson TJ, Klein TA, Gaffigan TV, Bergo E, Consolim J. Diagnosis by random amplified polymorphic DNA polymerase chain reaction of four cryptic species related to Anopheles (Nyssorhynchus) albitarsis (Diptera: Culicidae) from Paraguay, Argentina, and Brazil. J Med Entomol. 1995;32:697-704.

32. Li C, Wilkerson RC. Identification of Anopheles (Nyssorhynchus) albitarsis complex species (Diptera: Culicidae) using rDNA internal transcribed spacer 2based polymerase chain reaction primes. Mem Inst Oswaldo Cruz. 2005;100:495-500.

33. Brochero HL, Rey G, Buitrago LS, Olano VA. Biting activity and breeding sites of Anopheles species in the 
municipality of Villavicencio, Meta, Colombia. J Am Mosq Control Assoc. 2005;21:182-6.

34. Herrera S, Suárez $M$, Sánchez $G$, Quiñones $M L$, Herrera M. Uso de la técnica inmunoradiométrica (IRMA) en Anopheles de Colombia para la identificación de esporozoitos de Plasmodium. Colombia Med. 1987;18:2-6.

35. Collins W, Warren W, Skinner J, Sutton B. Infectivity of two strains of Plasmodium vivax to Anopheles albitarsis mosquitoes from Colombia. J Parasitol. 1985;71:771-3.

36. Quiñones ML, Suarez M, Fleming G. Estado de la susceptibilidad al DDT de los principales vectores de malaria en Colombia y su implicación epidemiológica. Biomédica. 1987;7:81-6.

37. Brochero HL. Estructura genética poblacional del mosquito Anopheles (Nyssorhynchus) marajoara
Galvão \& Damasceno 1942 (Diptera: Culicidae) de Colombia [tesis]. Bogotá, D.C.: Pontificia Universidad Javeriana; 2006. p.192.

38. Brochero H, Li C, Wilkerson RC. A newly recognized species in the Anopheles (Nyssorhynchus) Albitarsis Complex (Diptera: Culicidae) from Puerto Carreño, Colombia. Am J Trop Med Hyg. 2007;76:1113-7.

39. Sallum M, Wilkerson R, Forattini O. Taxonomic study of species formerly identified as Anopheles mediopunctatus and resurrection of An. costai (Diptera: Culicidae). J Med Entomol. 1999;36:282-300.

40. Wilkerson RC, Mureb Sallum MA. Anopheles (Anopheles) foratinii: a new species in series Arribalzagia (Diptera: Culicidae). J Med Entomol. 1999;36:345-54. 